\begin{tabular}{|c|c|c|}
\hline \multirow[t]{2}{*}{7} & $\begin{array}{l}\text { International Journal of Current Research in } \\
\text { Biosciences and Plant Biology }\end{array}$ & \\
\hline & Volume $4 \bullet$ Number 8 (August-2017) • ISSN: 2349-8080 (Online) & \\
\hline $\begin{array}{l}\text { EXCELLENT } \\
\text { PUBLISHERS } \\
\end{array}$ & Journal homepage: www.ijcrbp.com & wwwijecrip ocom \\
\hline
\end{tabular}

\title{
Measurement and Wireless Data Transmission of Heart Rate Using Pulse Sensor
}

\author{
Avvaru Srinivasulu* \\ Department of Electronics and Instrumentation Engineering, GITAM University, Bangalore, Karnataka- 561 203, India \\ *Corresponding author.
}

\begin{tabular}{|c|c|}
\hline Abstract & Article Info \\
\hline \multirow{8}{*}{$\begin{array}{l}\text { There is often a need to access heart rate measuring system which provides accurate and } \\
\text { quick readings of patients. This paper presents the design of simple heart rate } \\
\text { measurement system based on Arduino board which displays and simultaneously } \\
\text { transmitting the data to a smartphone via Bluetooth. Here the cardiac activity of the } \\
\text { patient is acquired by a pulse sensor and the output of this sensor is transmitted using } \\
\text { Bluetooth. By using this system unlike the conventional method, the doctor doesn't need } \\
\text { to be present at the time of measuring the heart rate. The system takes the physical input } \\
\text { from pulse sensor by placing the patients' finger on the sensor and then the input is } \\
\text { processed by Arduino to count the number of pulses and displaying the output in the } \\
\text { smartphone using a Bluetooth application. }\end{array}$} & $\begin{array}{l}\text { Accepted: 02 May } 2017 \\
\text { Available Online: } 06 \text { August } 2017\end{array}$ \\
\hline & Keywords \\
\hline & Arduino \\
\hline & Bluetooth \\
\hline & Cardiac activity \\
\hline & Heart rate \\
\hline & Pulse sensor \\
\hline & Smartphone \\
\hline
\end{tabular}

\section{Introduction}

The number of times the heart beats per minute is called heart rate or pulse. Normally, heart rate varies from person to person and it is an important heart-health gauge. As the age changes, the heart rate and regularity of pulse will be changing. That will signify the condition of heart. Generally, the heart rate can be found in a simple way that by [15] placing the tips of the index, second and third fingers on the palm side of another wrist below the base of the thumb or by placing the tips of the index and second fingers on the lower neck on either side of the windpipe. By Pressing and moving up or down lightly with fingers until we feel the blood pulsing beneath the fingers and counting the beats for 10 seconds and multiplying the number by six will give the beats per minute, nothing but heart rate. The doctor will suggest, to monitor the heart rate when the patient is on a beta blocker to decrease the heart rate or to control an arrhythmia [16]. Normally, the adult resting heart rate is 60-100 beats per minute (and children's is 70-100 for the age 6-15) [15]. This heart rate will not be same for the same person throughout a day. Based on the physical activities, the heart rate will change. If the activity demands more energy, the pulse will increase. When the exercise will be stopped, the pulse will not immediately return to normal. It gradually returns to its resting level. The sooner pulse rate will return to normal when the fitness level is greater [16]. When the person is frightened or surprised, due to the adrenaline response the heart rate will rise significantly. Some experienced athletes may see their resting heart rate fall below 60 
beats per minute. According to American Heart Association, heart rate during exercise is around 220 minus the person's age. The heart rate is a primary element to detect heart diseases. If the heart rate is above 100 beats per minute, it refers to Tachycardia (too fast at rest) [16]. If the heart rate is below 60 beats per minute, it refers to Bradycardia (too slow) [16]. Therefore, a design is presented in this paper to measure and transmit the heart rate.

\section{Existing methods}

There are number of designs proposed by different people [1-4][10-14]. Some of them are explained below.

Nazarene et al. [10] described the design of a measurement and monitoring of electrocardiogram (ECG) using three leads. The ECG signal was acquired from sticking gel $\mathrm{Ag} / \mathrm{AgCl}$ electrodes which were connected to the patient right and left arms and the third electrode was connected to right leg as a reference. The rest of the system consists of instrumentation amplifier and filter circuits etc., which were used for signal conditioning of the pulse input from the patient's body and displayed on CRO as the ECG waveform. Further, the signal was processed by the microcontroller AT89S52 to count the heart beat for one minute of duration. Finally, the heart rate was displayed on LCD. In this design, wireless transmission of the data was not established. Souvik Das [14] implemented a design of heart rate counter using a microcontroller (PIC series). This design was able to capture the pulse from fingertip by sensing the change in blood volume. A study was also done using the designed device and a standard heart rate measuring device for heart rate measurement on fifteen healthy normal subjects of age 21-22 yrs, both in relaxed and excited states. The results of the designed device were satisfactory. But the wireless communication was not provided in this design. Nisha Singh and Ravi Mishra [13] proposed a simple wireless transmission System using the wireless based Patient Sensor platform (WSP, Sensor Node) which has remote access capability. A prototype had been established based on a wireless communication channel (Wireless LAN (IEEE .802.15.4)). Desire sensor information (vital parameter) can be viewed remotely, and also vital parameter can be adjusted to meet demand (Standard sensor node (System on module) with common software). But the design is not implanted in real time.

Therefore our proposed method gives the implementation of the design for measuring the heart rate using pulse sensor and wireless communication is provided to the mobile phone through Bluetooth.

\section{Proposed method}

A Pulse sensor (Heart Beat sensor) and a Bluetooth module are connected to Arduino board. The Arduino board is programmed using Arduino sketch to get the readings and the Bluetooth module is interfaced to it. Now the data of heart rate per minute is sent to user's mobile unit via BT Simple Terminal application. The heart rate per minute can be seen from the mobile unit.

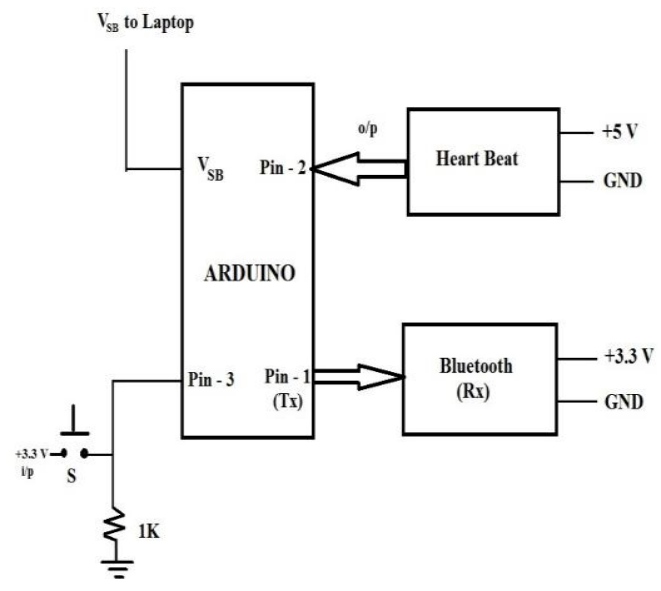

Fig. 1: Block diagram of proposed method.

\section{A. Principle of pulse sensor}

The one side of the sensor is having a LED and LDR setup [5,6] where the forefinger can be placed to capture the pulses. When the forefinger is placed in the middle of

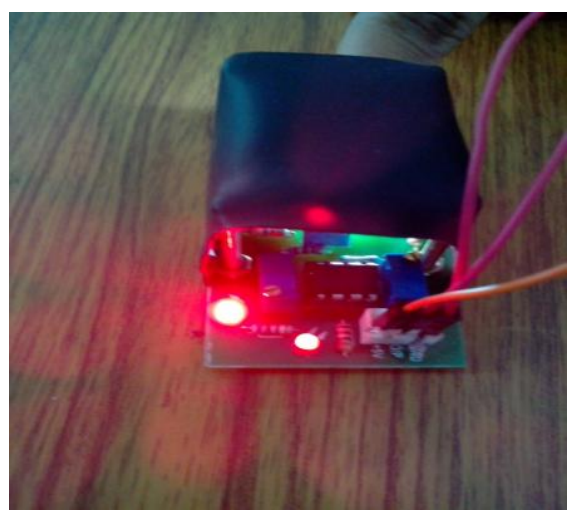

Fig. 2: Pulse sensor.

LED and LDR combination, based on the blood flow, the intensity of light reaching the LDR from LED will 
change. This will cause to capture the pulses which resemble the heart beat. There is a LED placed to indicate the heartbeat for every pulse detection. The same data is sent to the mobile through Bluetooth.

\section{B. Arduino Uno Smd R3}

The Arduino Uno [7-9] is an ATmega 328 microcontroller board. It has 14 digital input/output pins of which 6 can be used as PWM outputs, 6 analog inputs, a USB connection, a $16 \mathrm{MHz}$ crystal oscillator, an ICSP header, a power jack and a reset button. It contains everything needed to support the microcontroller to connect it to a computer through a USB cable and power it with an AC-to-DC adapter or battery to get started. It comes with an inbuilt boot loader to upload a new code to the Arduino Uno board, thus it does not need any external hardware programmer. Moreover, board can also be programmed by by-passing the boot loader and controlling the microcontroller using ICSP (In Circuit Serial Programming) Header.

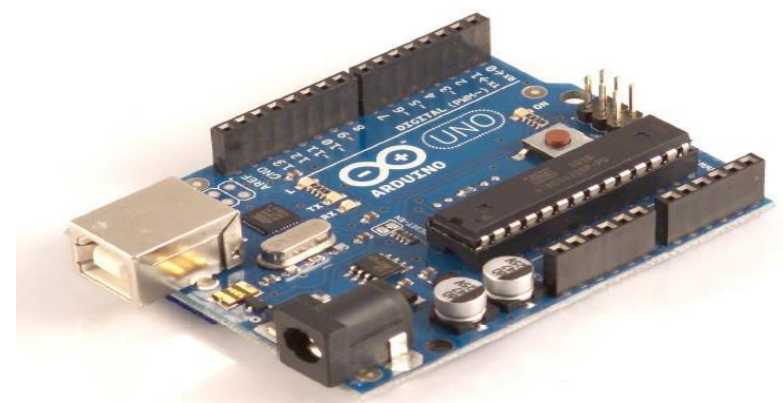

Fig. 3: Arduino uno.

\section{Bluetooth module}

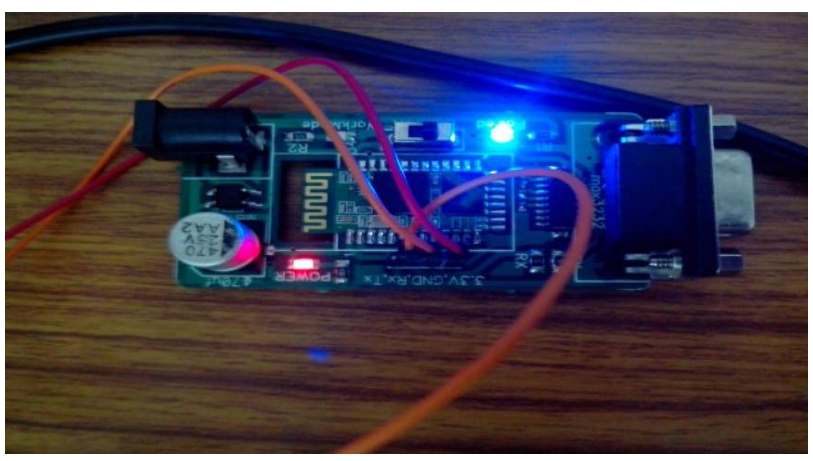

Fig. 4: HC-05 Bluetooth module.

It is needed to interface a Bluetooth module to the Arduino board to transmit the data that is to be sent to the Bluetooth-enabled smartphone. Here, HC-05 Bluetooth Module was used which is shown in Fig. 4.

\section{Flow chart}

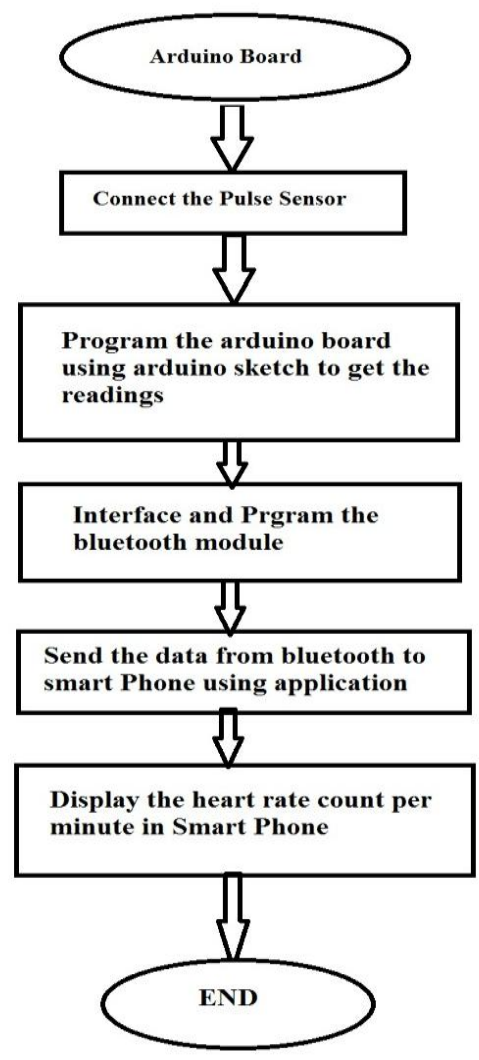

Fig. 5: Design flow chart.

\section{SOFTWARE}

\section{A. Ardiuno IDE}

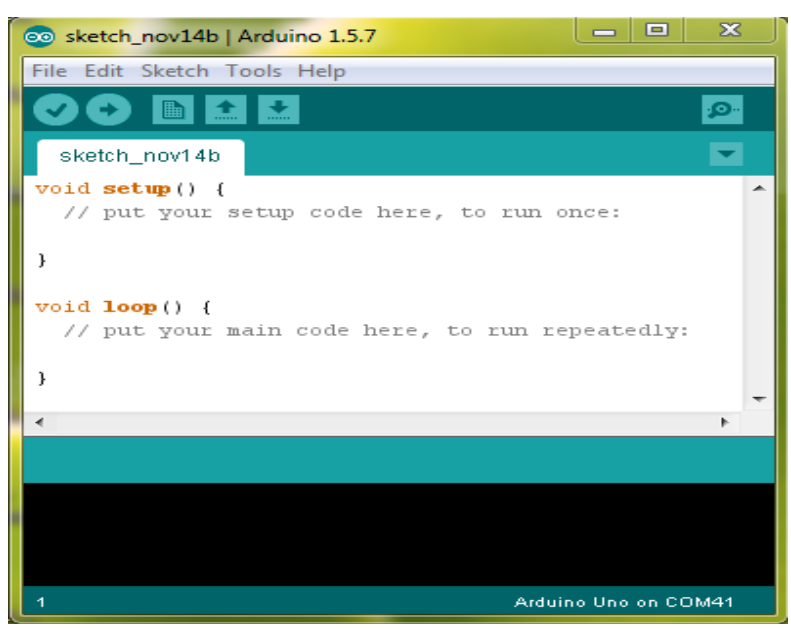

Fig. 6: Ardiuno IDE.

It provides an integrated development environment for programming of Arduino boards. A program or a code written for Arduino is called a SKETCH. Arduino sketch window looks like the one shown in Fig. 6. 


\section{B. Program code}

It is an open source development tool for writing programs on other computers. It is useful when we need the computers to talk to Arduino. Here we have used this to program an embedded $\mathrm{C}$ based application.

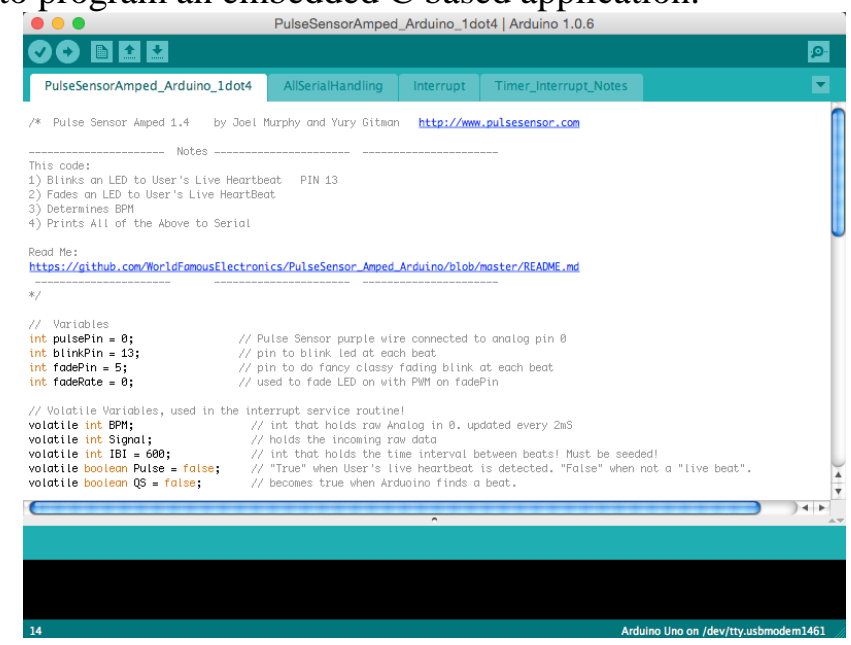

Fig. 7: Ardiuno IDE with program code.

\section{Bluetooth terminal application}

A simple Bluetooth communication app used to transmit / receive data from a compatible paired device. Sends logs from the terminal window via email for data transport. This app is perfect to use with the Real term, HyperTerminal, and Arduino.

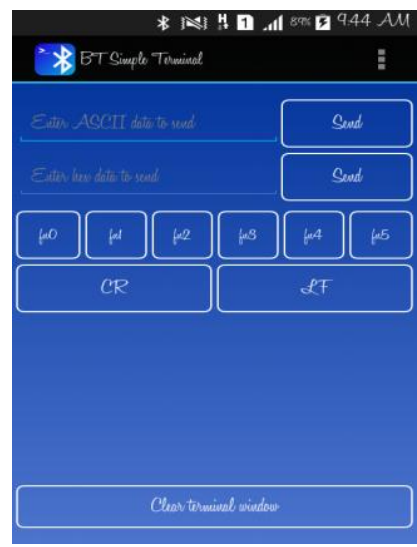

Fig. 8: Bluetooth terminal application.

\section{Features of proposed design}

The important features of this proposed system are,

- The Bluetooth technology is provided in almost all electronic gadgets like mobile phones, tablets, laptops, etc. So, we can use the personal mobile phone as a receiver end.
- This system can be implemented even in remote (mobile) patients.

- This system can be easily implemented in rural areas as it is cost effective.

\section{Results}

Once the forefinger is placed at the correct position on the pulse sensor, the device starts to measure the heart rate and the information is sent to the smart phone via Bluetooth using a Bluetooth application. And for every beat, the message is displayed on the mobile screen. This will continue up to one minute. Finally, after one minute, the device stops to record and send the beats to the mobile phone. The last message that has been displayed is the number of beats per minute. The heart rate sent to smartphone is displayed as shown in the figure below:

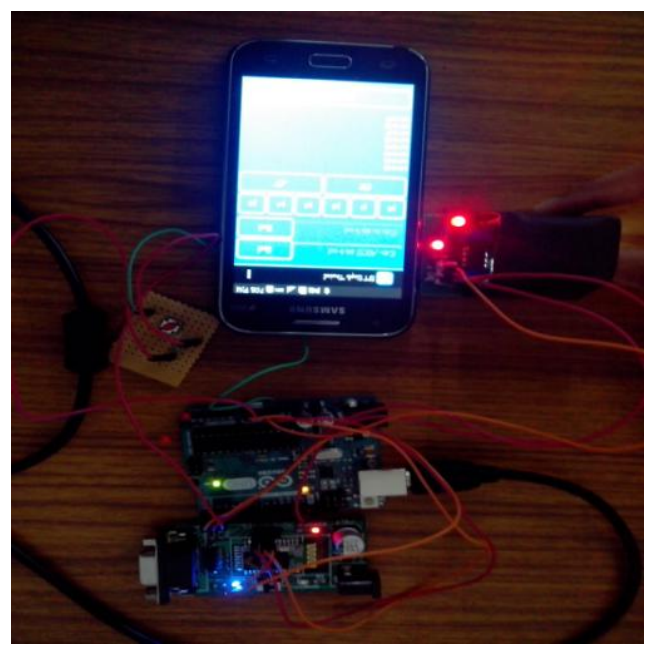

Fig. 9 (a): Experimental setup.

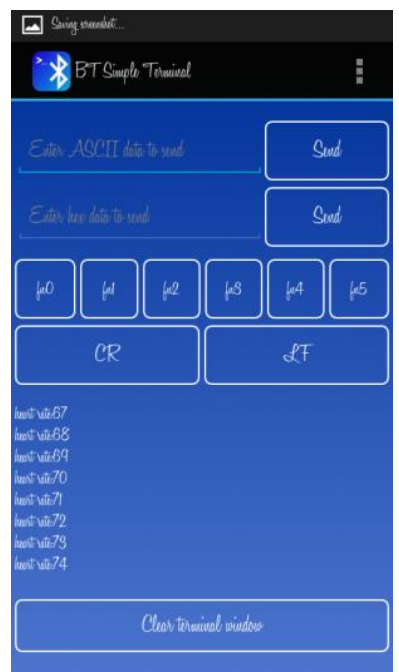

Fig. 9(b): Heart rate display on mobile. 


\section{Conclusion}

This paper presents the design of heart rate measurement and monitoring system using pulse sensor. The designed system also provided the heart rate measurement sending to the mobile phone through Bluetooth technology. This helps to provide wireless communication between patient and doctor or physician. As the designed system is cost effective and easy to use, this will reach easily to rural people. In future, the design can be extended by using wifi or GSM or GPS for long distance communication.

\section{Conflict of interest statement}

Authors declare that they have no conflict of interest.

\section{References}

[1] Samuel E. de Lucena, Daniel J. B. S. Sampaio, "ECG Monitoring Using Android Mobile Phone and Bluetooth" Instru and Measurement Tech Conference (I2MTC), IEEE International, pp.19761980, May 2015.

[2] Liang-Hung Wang, Tsung-Yen Chen, Shuenn-Yuh Lee, Tai-Hsuan Yan, Shi-Yan Huang, Jen-Hao W, and Kuang-Hao Lin , "A Wireless Electrocardiogram Detection for Personal Health Monitoring " Orange Tech-nologies (ICOT), International Conference,pp.43- 46, May 2013.

[3] http://www.emo.org.tr/ekler/a568a2aa8c19a31 ek. pdf.

[4] Prasad Kumari Nisha , Yadav Vinita, "Heart Rate Monitoring and Data Transmission via Bluetooth",
International Journal of Innovative and Emerging Research in Engineering, Volume 2, Issue 2, pp 99$105,2015$.

[5] http://pulsesensor.com

[6] http://pulsesensor.com/collections/frontpage/ products / pulse-sensor-amped

[7] http://arduino.cc/en/Main/ArduinoBoardUno

[8] http://rhydolabz.com

[9] http://processing.org

[10] Naazneen M.G., Sumaya Fathima, Syeda Husna Mohamaddi, Sarah Iram L .Indikar, Abdul Saleem, Mohamed Jebran, "Design and Implementation of ECG Monitoring and Heart Rate Measurement System", International Journal of Engineering Science and Innovative Technology IJESIT, Volume 2 ,Issue 3, May 2013.

[11] Lee H., Park S., and Woo E., "Remote Patient Monitoring Through the World-Wide Web," in Proceedings of the 19th International Conference of IEEE, Chicago, IL, USA, pp. 928-931, 1997.

[12] Y. Hao and J. Foster, "Wireless sensor networks for health monitoring applications," Physiological Meas., vol. 29, no. 11, pp. R27-R56, 2008.

[13] Nisha singh and Ravi Mishra, "Microcontroller Based Wireless Temperature And Heart Beat ReadOut", IOSR Journal of Engineering (IOSRJEN), Vol. 3, Issue 1, PP 01-06, Jan 2013.

[14] Souvik Das, "The Development of a Microcontroller Based LowCost Heart Rate Counter for Health Care Systems", International Journal of Engineering Trends and Technology, Volume4,Issue2, pp 207-211, 2013.

[15] http://my.clevelandclinic.org

[16] http://www.heart.org

\section{How to cite this article:}

Srinivasulu, A., 2017. Measurement and wireless data transmission of heart rate using pulse sensor. Int. J. Curr. Res. Biosci. Plant Biol. 4(8), 42-46. doi: https://doi.org/10.20546/ijcrbp.2017.408.006 